\title{
A Meshfree Method for Deformation Field Reconstruction of Soft Tissue in Needle Insertion
}

\author{
Jingtao Chen* \\ Shenzhen Institutes of Advanced \\ Technology, Chinese Academy of \\ Sciences, Shenzhen, China \\ jt.chen1@siat.ac.cn
}

\author{
Zeng Lin* \\ Shenzhen Institutes of Advanced \\ Technology, Chinese Academy of \\ Sciences, Shenzhen, China \\ zeng.lin@siat.ac.cn
}

\author{
Shoujun $\mathrm{Zhou}^{\dagger}$ \\ Shenzhen Institutes of Advanced \\ Technology, Chinese Academy of \\ Sciences, Shenzhen, China \\ sj.zhou@siat.ac.cn
}

\author{
Tiexiang Wen \\ National Innovation Center for \\ Advanced Medical Devices, Shenzhen, \\ China \\ wentiexiang@nmed.org.cn
}

\author{
Quan Zeng \\ Shenzhen Institutes of Advanced \\ Technology, Chinese Academy of \\ Sciences, Shenzhen, China \\ quan.zeng@siat.ac.cn
}

\section{CCS CONCEPTS}

\begin{abstract}
Objective: The deformation field inside the soft tissue is useful to predict and track the specific target of needle insertion. Finite element (FE) provides a sensorless way to reconstruct the deformation field inside soft tissue. However, the time-consuming model meshing makes it difficult to automate the reconstruction during needle insertion operation. The purpose of this work is to present a numerical method that can automatically reconstruction of deformation field of large-deformed soft tissue during needle insertion. Methods: Reproducing kernel particle method (RKPM) was used to reconstruct the deformation and stress field of soft tissue with real-time acquired displacement and force boundary conditions. The tissue crack was simulated by employing a node split mechanism. The validation experiment involves puncturing a silicone phantom with a robotic arm integrated with a needle. Results: The reconstructed displacements approach the experimental measurements with the average error of $0.15 \mathrm{~mm}, 0.30 \mathrm{~mm}, 0.63 \mathrm{~mm}$, and $0.55 \mathrm{~mm}$ respectively at $12 \mathrm{~mm}, 24 \mathrm{~mm}, 36 \mathrm{~mm}$, and $40 \mathrm{~mm}$ insertion depths. The reconstructed data have respectively $88.9 \%, 50 \%, 16.7 \%$, and $27.8 \%$ nodes with an absolute error of less than $0.3 \mathrm{~mm}$ ( 2 pixels). The stress relaxation of the silicon model has been revealed and be used to qualitatively explain the reconstruction error. Von-mises stress field has been also presented and registered into the X-ray image. Conclusion: The proposed meshfree-based method has acceptable accuracy for reconstructing the deformation field inside the large-deformed organ.
\end{abstract}

\footnotetext{
*Jingtao Chen and Zeng Lin contributed equally to this study and share first authorship. ${ }^{\dagger}$ Address correspondence to: Shoujun Zhou, 1068 Xueyuan Avenue, Shenzhen Univer-
} sity Town, Shenzhen, GD 518055, P.R. China.

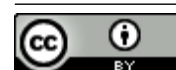

This work is licensed under a Creative Commons Attribution International 4.0 License.

ICBBS 2021, October 29-31, 2021, Xiamen, China

(C) 2021 Association for Computing Machinery.

ACM ISBN 978-1-4503-8430-8/21/10.

https://doi.org/10.1145/3498731.3498738

\section{KEYWORDS}

- Computing methodologies $\rightarrow$ Modeling and simulation.

meshfree method, needle insertion, computational biomechanics, soft tissue deformation

\section{ACM Reference Format:}

Jingtao Chen, Zeng Lin, Shoujun Zhou, Tiexiang Wen, and Quan Zeng. 2021. A Meshfree Method for Deformation Field Reconstruction of Soft Tissue in Needle Insertion. In 2021 10th International Conference on Bioinformatics and Biomedical Science (ICBBS 2021), October 29-31, 2021, Xiamen, China. ACM, New York, NY, USA, 9 pages. https://doi.org/10.1145/3498731.3498738

\section{INTRODUCTION}

Needle insertion into soft tissue is an important technique in extracting biopsy samples, placing radioactive seeds around tumors in brachytherapy [1], [2]. For the former, accurate needle insertion to the preoperative planned sampling area is very important for tumor diagnosis [ref]; for the latter, the accuracy of the needle insertion determines whether the tumor is inside the radiation range which is crucial to the effect of the therapy [3]. Inaccurate needle insertion increases the trauma of the patient and affects the effect of the operation. The deformation of soft organs is an important reason for inaccurate needle insertion [4], [5].

The real-time deformation field inside the soft tissues could be useful for predicting and tracking the specific target during organ deformation. Biomechanical modeling provides a sensorless way to reconstruct the deformation field of soft tissues. The FE and meshfree methods are two streams that have been currently used in the soft tissue deformation simulation [6]. The former is most frequently be applied in infinitesimal deformations problems. The early FE method, especially for the most frequently used lower order linear shape functions, has limitations mainly caused by two basic assumptions: linear elastic material and infinitesimal deformation. As needle insertion into an organ is a non-linear problem of continuum mechanics which involves large deformations and large strains with non-linear material, and the FE method is incompatible with the assumption of infinitesimal deformations [7]. To improve 
the computation accuracy, different computation models including the co-rotational finite elements [8], [9], and the Total Lagrangian explicit dynamics algorithm [10-12] have been proposed. Although both can be applied in real-time deformation computation, they are still based on the theory of small deformation and linear material properties. Besides that, the accuracy of those FE methods relies extremely on high-quality mesh which requires organ image segmentation, geometry model surface smoothing, and meshes fining [13], [14]. These preliminary modeling tasks are very laborintensive and time-consuming which makes it difficult to automate the computation during the needle insertion procedure.

To overcome the main challenges brought by the finite element algorithm, the meshfree-based method has been proposed as it avoids the model meshing work. Meshfree (or meshless) method is a collective name that covers a class of particle methods [15]. Differing with the mesh-dependent numerical methods, the shape functions of meshfree methods are set up at the nodes or particles, i.e., the construction of meshfree shape functions doesn't rely on the mesh between adjoining nodes. These characteristics make meshfree methods are more suitable for engineering and mechanical problems, such as organ deformation [7], elastic damage analysis [16], soft tissue damage [17], impact failure modeling [18], crack propagation [19], and so on. The remarkable successes in the real world's application also promoted the study to the meshfree methods themselves. Many researchers devoted to the developments of various Galerkin meshfree methods, for instance, diffuse element method [20], element free Galerkin method [21], [22], reproducing kernel particle method [23], h-p cloud method [24], partition of unity method [25], and others. Otherwise, some collocation methods based on meshfree shape functions have also been proposed, for example, radial basis collocation method [26], [27], local radial basis function collocation method [28], reproducing kernel enhanced local radial basis collocation method [29], finite point method [30], gradient smoothing meshfree collocation method [31], etc. Comparing with the $C^{0}$ continuous finite element shape functions, the meshfree methods are easier to construct arbitrary high order smooth shape functions and have higher numerical accuracy.

As the advantages of the meshfree method in computing large deformation of soft tissue, many researchers started to apply the meshfree method into the surgical simulation. Joldes et al. proposed a meshless method based on complete Lagrangian explicit dynamics (MTLED) [32] and applied it to simulate the large deformation of brain drift in 3D [7]. Even if the imposition of the essential boundary condition has been improved, but it only simulated soft tissue deformation in the indentation. Rausch et al. [17] proposed a meshless method based on smooth particle hydrodynamics (SPH) and simulated the stress in the normal and glycosaminoglycan accumulation area on the thoracic aorta [33]. According to the respiratory management strategy, doctors generally ask the patient to hold their breath during needle insertion [34]. Therefore, the insertion force applied on the inserted organ exerted by the needle is the main cause of deformation. The extended element-free Galerkin method (XEFG) has been also proposed for modeling displacement and stress fields in cuts/cracks [35]. Jin et al. [36] proposed a meshless total Lagrangian adaptive dynamic relaxation (MTLADR) method to modeling the topology change of nodes in surgical cutting. However, the method was validated by comparing with commercial FE code instead of experiment results. Wittek et al. [37] has employed the MTLED method to predict the displacement field during needle insertion into a silicone model at depth until $15 \mathrm{~mm}$.

This paper aims to reconstruct the displacement field of soft tissue during a computer-assisted needle insertion procedure. For meeting the real-time and automated computation purpose, an RKPM-based meshfree method was employed to reconstruct the displacement and stress field by integrating boundaries condition obtained in a real-time manner and automatic update of nodes. The tissue crack/cutting and its viscoelasticity were considered in the proposed meshfree method. To accelerate the computation speed, we focus on solving the $2 \mathrm{D}$ deformation of the silicon model in this work.

The layout of this paper is as follows. An RKPM-based meshfree method for reconstructing the deformation fields was proposed in Section 2. To validate the proposed method, a needle insertion experiment that involves using the robotic arm to penetrate a silicon model integrated with metallic beads was presented in Section 3. Finally, the reconstructed results and experimental measurements have been compared, and the stress relaxation of the silicon model was discussed in Section 4.

\section{MATERIALS AND METHODS}

\subsection{Experiment Method}

A model made by SYLGARDTM 572 silicone dielectric gel was used as the needle insertion target (Figure 1. A). The silicon model was solidified and fixed inside a 3D-printed cuboid container. The metallic beads were integrated inside for detecting the displacement (Figure 1. B). Two layers of metallic beads with $1 \mathrm{~mm}$ (upper layer) and $0.6 \mathrm{~mm}$ (lower layer) diameters are respectively distributed on two layers inside the silicon model (Figure 1. B). As they are very small relative to the entire silicon model, it is still considered to be homogenous.

A UR3e robotic arm integrating a percutaneous transhepatic cholangiography needle at the end $(\mathrm{d}=1.2 \mathrm{~mm})$, was controlled to puncture down at a fixed speed $(2 \mathrm{~mm} / \mathrm{s})$ for 20 seconds. A SIEMENS ARCADIS ORBIC 3D C-Arm X-ray machine was used to record the trajectories of the metallic beads and needle during the insertion process. To guarantee the consistency of boundary conditions between the experiment and simulation/reconstruction, a force sensor (SIMBATOUCH SBT740-5KG) was used to record the needle insertion force and send the data to the computer in real-time (Figure 1. C).

\subsection{Numerical method}

The RKPM was used to reconstruct the deformation and stress field during needle insertion. The needle is assumed to be a rigid body that inserts toward down. As the needle did not deflect, the crack propagates along the needle insertion direction. The crack of the silicon model caused by needle insertion was simulated by splitting nodes. To investigate the influence of nodes number on reconstruction accuracy, the different number of nodes $(836,1881$, and 3344 nodes) were respectively used in the reconstruction. The needle insertion force detected by the force sensor and the displacement constraints of crack are respectively used as the traction force boundary and displacement boundaries. 


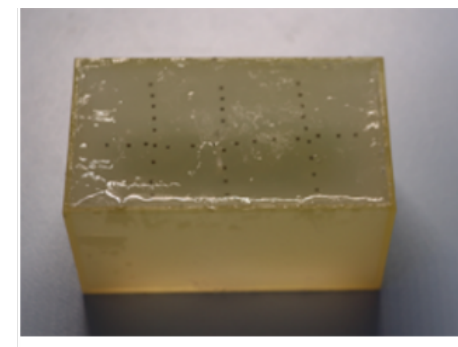

A

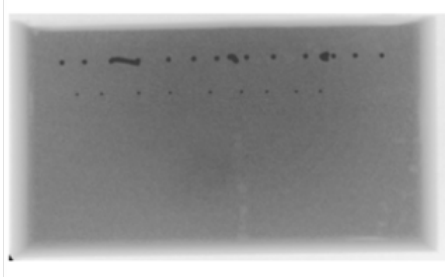

$\mathrm{B}$

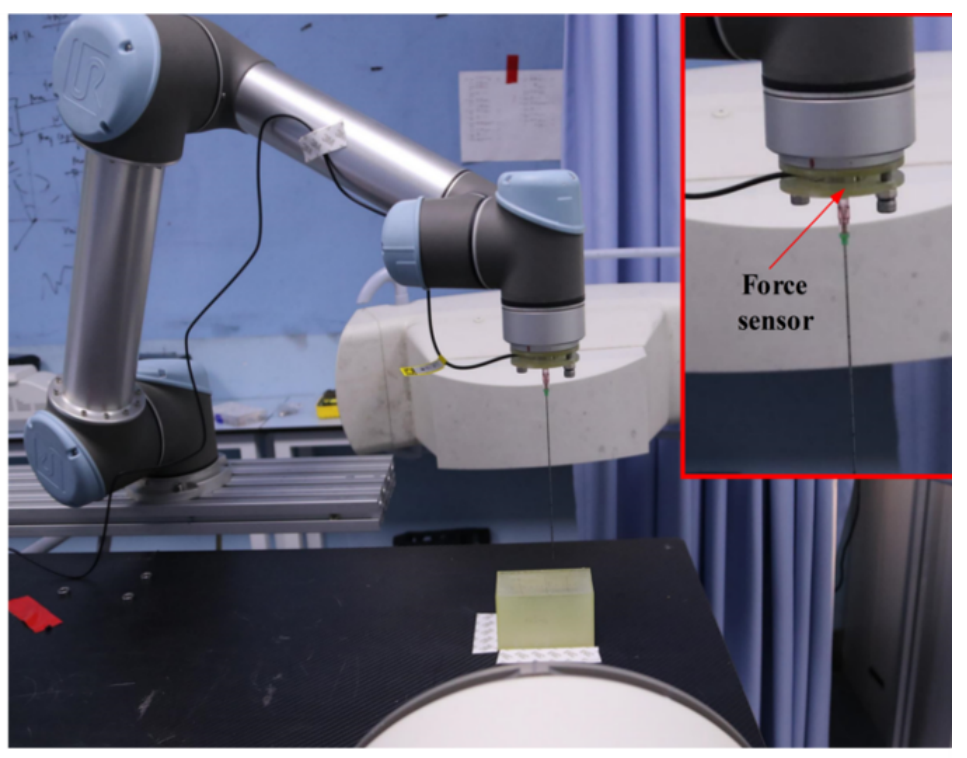

$\mathrm{C}$

Figure 1: Needle insertion experiment setup: A. Silicon model (sizes $50 \mathrm{~mm} \times 90 \mathrm{~mm} \times 50 \mathrm{~mm}$ ) integrated with metallic beads; B. $\mathrm{X}$-ray image of silicon model with two layers of metallic beads; C. Experiment scenario showing the robotic arm integrated with needle and force sensor.

2.2.1 Governing equation. The whole dynamic simulation was driven by updating boundary conditions including needle tip position and needle insertion force in each iterative calculation. As the diameter of the needle is very small compared with the width of the silicon model, the simulation was carried out using discrete equations in two dimensions. Consider the following twodimensional problem on the domain $\Omega$ (the cross-section of silicon model) bounded by $\Gamma$ (the geometry boundary of the cross-section of silicon model including the crack), then the governing equation is:

$$
\nabla \cdot \sigma+\mathbf{b}=0 \operatorname{in} \Omega
$$

where $\sigma=\mathrm{C}: \varepsilon$ is the stress tensor, and the $\varepsilon=1 / 2(\nabla \mathbf{u}+\mathbf{u} \nabla)$ is the strain tensor. Here $\boldsymbol{u}$ is the displacement field, $\boldsymbol{b}$ is the needle insertion force vector. The boundary conditions are as follows:

$$
\begin{gathered}
\sigma \cdot \mathbf{n}=\overline{\mathbf{t}} \text { on } \Gamma_{t} \\
\mathbf{u}=\overline{\mathbf{u}} \text { on } \Gamma_{u}
\end{gathered}
$$

where $\overline{\mathbf{t}}$ is the prescribed traction force, i.e. needle force vector, on the loading boundary $\Gamma_{t}, \overline{\mathbf{u}}$ is the prescribed displacement boundaries value which evolves as needle inserting deeper on the boundary $\Gamma_{u}$.

Consider the trail function $\boldsymbol{u}(\boldsymbol{x}) \in H^{1}$ and Lagrange multipliers $\lambda \in H^{0}$ for all test functions $\delta \mathbf{v}(\mathbf{x}) \in H^{1}$ and $\delta \lambda \in H^{0}$, the weak form of the governing equation is posed as follows:

$$
\begin{array}{r}
\int_{\Omega} \delta\left(\nabla_{s} \mathbf{v}^{T}\right): \sigma d \Omega-\int_{\Omega} \delta \mathbf{v}^{T} \cdot \mathbf{b} d \Omega-\int_{\Gamma_{t}} \delta \mathbf{v}^{T} \cdot \overline{\mathbf{t}} d \Gamma \\
-\int_{\Gamma_{u}} \delta^{T} \cdot(\mathbf{u}-\overline{\mathbf{u}}) d \Gamma-\int_{\Gamma_{u}} \delta \mathbf{v}^{T} \cdot \lambda d \Gamma=0
\end{array}
$$

Here $\nabla_{s} \boldsymbol{v}^{T}$ is the symmetric part of $\nabla \boldsymbol{v}^{T} ; H^{1}$ and $H^{0}$ denote the Sobolev space of degree one and zero. It should be mentioned here since the reproducing kernel particle meshfree shape functions do not satisfy the interpolation property, the Lagrange multipliers method is adopted to assemble the essential boundary conditions.

2.2.2 Reproducing kernel particle method. The reproducing kernel approximation of a field variable $u(\mathbf{x})$, denoted by $u^{h}(\mathbf{x})$, can be expressed as:

$$
u^{h}(x)=\sum_{I=1}^{N P} \Psi_{I}(x) d_{I}
$$

where $d_{I}$ is the nodal coefficient associated with the node $\mathbf{x}_{I}$. $\Psi_{I}(x)$ is the reproducing kernel shape function that takes the following formula:

$$
\Psi_{I}(x)=c\left(x_{I}-x ; x\right) \phi_{s}\left(x_{I}-x\right)
$$

in which $c\left(\mathbf{x}_{I}-\mathbf{x} ; \mathbf{x}\right)$ is the correction function and the non-negative kernel function $\phi_{S}\left(\mathbf{x}_{I}-\mathbf{x}\right)$ located at $\mathbf{x}_{I}$ has a support to define the nodal influence domain $\operatorname{supp}\left(\mathbf{x}_{I}\right)$, i.e., $\phi_{S}\left(\mathbf{x}_{I}-\mathbf{x}\right)$ will vanish when $\left\|\mathbf{x}_{I}-\mathbf{x}\right\| \geq s$. In this study, the following cubic B-spline function

$$
\phi(v)= \begin{cases}\frac{2}{3}-4 v^{2}+4 v^{3}, & v \leq \frac{1}{2} \\ \frac{4}{3}-4 v+4 v^{2}-\frac{4}{3} v^{3}, & \frac{1}{2}<v \leq 1 \\ 0, & v>1\end{cases}
$$

is used as the kernel function.

For the purpose of reproducing polynomial exactly, $c\left(\mathbf{x}_{I}-\mathbf{x} ; \mathbf{x}\right)$ can be expressed to the formulation:

$$
c\left(\mathbf{x}_{I}-\mathbf{x} ; \mathbf{x}\right)=\mathbf{p}^{T}\left(\mathbf{x}_{I}-\mathbf{x}\right) \mathbf{a}(\mathbf{x})
$$


where $\mathbf{a}(\mathbf{x})$ is the coefficients vector to be determined, $\mathbf{p}(\mathbf{x})$ is the $p$-th order monomial basis vector:

$\mathbf{p}(\mathbf{x})=\left\{1, x_{1}, x_{2}, \cdot s, x_{n_{s d}}, x_{1}^{2}, x_{1} x_{2}, \cdot s, x_{n_{s d}}^{2}, \cdot s, x_{1}^{p}, x_{1}^{p-1} x_{2} \cdot s, x_{n_{s d}}^{p}\right\}^{T}$

where $n_{s d}$ denotes the spatial dimension. The position-dependent unknown vector $\mathbf{a}(\mathbf{x})$ in (6) is solved by enforcing the $p$-th order consistency conditions:

$$
\sum_{I=1}^{N P} \Psi_{I}(\mathbf{x}) \mathbf{x}_{I}^{\alpha}=\mathbf{x}^{\alpha},|\boldsymbol{\alpha}| \leq p
$$

For convenience in expressions, the multi-index notations are used herein, i.e., $\boldsymbol{\alpha}=\left(\alpha_{1}, \cdot s, \alpha_{n_{s d}}\right),|\boldsymbol{\alpha}|=\alpha_{1}+\cdot s+\alpha_{n_{s d}}$. Eq. (10) can be recast to the vector formula:

$$
\sum_{I=1}^{N P} \Psi_{I}(\mathbf{x}) \mathbf{p}\left(\mathbf{x}_{I}\right)=\mathbf{p}(\mathbf{x})
$$

or equivalently,

$$
\sum_{I=1}^{N P} \Psi_{I}(\mathbf{x}) \mathbf{p}\left(\mathbf{x}_{I}-\mathbf{x}\right)=\mathbf{p}(\mathbf{0})
$$

Substituting (6) into (12) immediately gives:

$$
\mathrm{A}(\mathrm{x}) \mathrm{a}(\mathrm{x})=\mathrm{p}(\mathbf{0})
$$

where $\mathbf{A}(\mathbf{x})$ is the moment matrix defined as:

$$
\mathbf{A}(\mathbf{x})=\sum_{I=1}^{N P} \mathbf{p}\left(\mathbf{x}_{I}-\mathbf{x}\right) \mathbf{p}^{T}\left(\mathbf{x}_{I}-\mathbf{x}\right) \phi_{s}\left(\mathbf{x}_{I}-\mathbf{x}\right)
$$

Thus we obtain $\mathbf{a}(\mathbf{x})=\mathbf{A}^{-1}(\mathbf{x}) \mathbf{p}(\mathbf{0})$ from (13) and the reproducing kernel shape function of Eq. (6) then becomes:

$$
\Psi_{I}(\mathbf{x})=\mathbf{p}^{T}(0) \mathrm{A}^{-1}(\mathbf{x}) \mathbf{p}\left(\mathbf{x}_{I}-\mathbf{x}\right) \phi_{S}\left(\mathbf{x}_{I}-\mathbf{x}\right)
$$

Through directly computing, it is easy to get the first-order derivative of the shape function:

$$
\begin{gathered}
\Psi_{I, x}(\mathbf{x})=\mathbf{p}^{T}(\mathbf{0})\left[\mathbf{A}_{, x}^{-1}(\mathbf{x}) \mathbf{p}\left(\mathbf{x}_{I}-\mathbf{x}\right) \phi_{S}\left(\mathbf{x}_{I}-\mathbf{x}\right)\right. \\
+\mathbf{A}^{-1}(\mathbf{x}) \mathbf{p}, x\left(\mathbf{x}_{I}-\mathbf{x}\right) \phi_{S}\left(\mathbf{x}_{I}-\mathbf{x}\right) \\
\left.+\mathbf{A}^{-1}(\mathbf{x}) \mathbf{p}\left(\mathbf{x}_{I}-\mathbf{x}\right) \phi_{S, x}\left(\mathbf{x}_{I}-\mathbf{x}\right)\right]
\end{gathered}
$$

in which $\mathrm{A}_{, x}^{-1}(\mathbf{x})$ is derived from the relationship of $\mathrm{AA}^{-1}=\mathbf{I}$ :

$$
\mathrm{A}_{, x}^{-1}(\mathbf{x})=-\mathrm{A}^{-1}(\mathbf{x}) \mathbf{A}_{, x}(\mathbf{x}) \mathbf{A}^{-1}(\mathbf{x})
$$

2.2.3 Node split mechanism. The topology changes of soft tissue caused by needle insertion are modeled by splitting nodes along the insertion path. The cutting-induced discontinuity is modeled solely through changes in the nodal domain of influence. The effect of needle insertion is reflected in the changes of the shape and size of the influence domain. The shape of the influence domain used here is a rectangle. Based on the level set method proposed by Jin et al [36], the effect of the needle insertion on the size of the influence domain was realized in this way: if the connection line between two nodes intersects with the needle shaft, then they should be eliminated from the influence domain of each other. Since the relative position of the needle tip inside the organ can be given by the robotic arm, the node to be split can be detected. The original node will be shifted to the left side of the needle shaft while the newly added node shifts to the right. Consequently, the original and the split node together form the new geometry boundaries (Figure 2).

2.2.4 Boundary conditions. To verify the accuracy of the proposed meshfree method, it is necessary to keep the consistency between the boundary conditions of simulation and the experiment including the displacement constrain and the traction force. As the silicon model was installed inside a cuboid box (as shown in Figure 1A), the nodes located on the left, right, and bottom of the geometry model has no displacement. Same as human soft organs, the silicon model has viscoelasticity which makes nodes located on the crack move down with the needle shaft. As the silicone model used in the experiment is a homogeneous material, according to the shear flow dynamic equation with uniform velocity gradient, the shear stress $\tau$ can be expressed as:

$$
\tau=\mu \frac{d v}{d x}
$$

where $\mu$ is the viscosity coefficient; $x$ is the horizontal direction; $v(x)$ is the local average of silicon gel velocity and it is only the function of $x$. Hence the displacement of $i$ th node $D_{i}$ on the crack boundary at moment $t$ can be expressed as:

$$
D_{i}(t)=\int_{0}^{t} v_{i}(a) d t=\int_{0}^{t} a \frac{\tau_{i}(t)}{\mu} d t
$$

where $v_{i}(x)$ is the ith node velocity at $a$; $a$ is the horizontal distance between the node and left side of the silicone model; $\tau_{i}(t)$ is the shear stress at the $i$ th node which inherited from the calculation results of the last iterative step. Hence, the displacement constraints for the new boundaries induced by the crack at $k$ step was given as:

$$
\overline{u_{i}^{k}}=D_{i}(k)-D_{i}(k-1)
$$

The traction/needle insertion force boundary in each iterative step is determined as:

$$
\overline{t_{i}}=F_{i}
$$

where $F_{i}$ is the needle insertion force detected by the force sensor.

\subsection{Data analysis}

2.3.1 Experimental data. The positions of metallic beads during the process were recorded and their displacements were extracted to be compared with the reconstruction results. As limited by the imaging speed of the $\mathrm{C}$-arm machine, four X-ray images were captured when the needle was inserted into $12 \mathrm{~mm}, 24 \mathrm{~mm}, 36 \mathrm{~mm}$, and $40 \mathrm{~mm}$. A region-growing-based method was used to segment the metallic beads and consequently, their displacements were calculated by comparing them to their initial positions.

2.3.2 Reconstructed data. The displacement and stress field inside the silicon model during the needle insertion process were reconstructed using the proposed meshfree method. In order to validate the reconstructed data, the displacements of nodes corresponding to the actual metallic beads were calculated and be compared with experimental measurements. Due to the lack of an acceptable range of absolute error between the above two types of data, two different accuracy standards are used here: 2 or 3 pixels. As the resolution of the X-ray image is $0.15 \mathrm{~mm} \times 0.15 \mathrm{~mm}$, then the $2 \times 0.15 \mathrm{~mm}=0.3 \mathrm{~mm}$ and $3 \times 0.15 \mathrm{~mm}=0.45 \mathrm{~mm}$ are respectively used as the accuracy criterion for evaluating the reconstructed data. In 

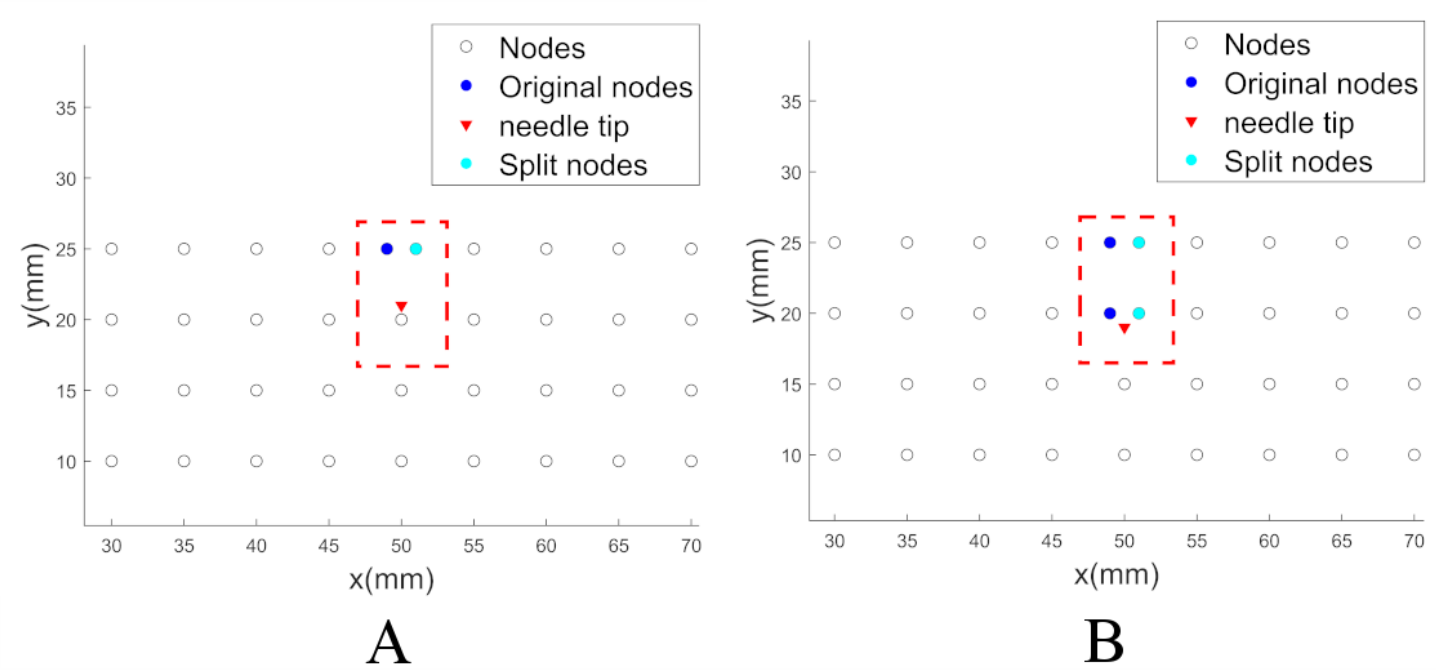

Figure 2: Node split mechanism. A. Needle tip being contacted with the node below; $B$. The contacted node splits into two nodes. The original point locates in the center point of the left side of the cross-section of the silicon model which is a rectangle. $X$ and $\mathrm{Y}$ axis represent respectively the $\mathrm{x}$ and $\mathrm{y}$ coordinates.

Section 3, the reconstructed stress fields inside the silicon model at the moment of capturing X-ray images are also presented.

\section{RESULTS}

The deformation field inside the silicon model during needle insertion was reconstructed by using the proposed RKPM-based meshfree method. The reconstructed results were validated against the measured displacements of 18 metallic beads.

\subsection{Reconstructed displacement versus experimental measurement}

3.1.1 Overall trend of displacement field. Figure 3 presents the comparison between the displacement of reconstructed data and the experimental measurements. As mentioned before, the displacements of each point at different insertion depths are obtained by calculating the difference between its current position and initial position. No.1-10 points are in the first layer distributed from left to right while the rest of them are in the second layer. The experimental measurements generally show two waves respectively correspond to the deformation of the metal points in two layers. Two similar waves are also found in the reconstructed displacements. The two largest peaks occur respectively on the closest points to the needle shaft in two layers of metallic beads. Comparing the results of using three different numbers of nodes $(836,1881$, and 3344 nodes), there are no evident differences among them. The reconstructed positions of the metallic beads were drawn in the same coordination as the experimental measurements in Figure 4. For the $1^{s t}, 2^{\text {nd }}, 3^{r d}$, and $4^{\text {th }} \mathrm{X}$-ray images, the insertion depths were respectively $12 \mathrm{~mm}, 24 \mathrm{~mm}, 36 \mathrm{~mm}$, and $40 \mathrm{~mm}$ taken at $t_{1}=6 \mathrm{~s}, t_{2}=12 \mathrm{~s}$, $t_{3}=18 \mathrm{~s}$, and $t_{4}=20 \mathrm{~s}$.

3.1.2 Absolute error. The absolute errors between the reconstructed and experimental data at insertion depths $12 \mathrm{~mm}, 24 \mathrm{~mm}$,
$36 \mathrm{~mm}$, and $40 \mathrm{~mm}$ were presented in Figure 5. As the increase of nodes doesn't influence the reconstruction results significantly, only the absolute errors with using 1881 nodes were reported here. The reconstructed data have respectively $88.9 \%, 50 \%, 16.7 \%$, and $27.8 \%$ nodes with an absolute error of less than $0.3 \mathrm{~mm}$ ( 2 pixels) at four insertion depths from $12 \mathrm{~mm}$ to $40 \mathrm{~mm}$. With changing the accuracy criteria to $0.45 \mathrm{~mm}$ ( 3 pixels), those rates increase respectively to $100 \%, 83.3 \%, 27.8 \%$, and $38.9 \%$. The average values of the absolute error vary from $0.15 \mathrm{~mm}, 0.30 \mathrm{~mm}, 0.63 \mathrm{~mm}$, and $0.55 \mathrm{~mm}$ as the insertion depth increase, and the total average value for absolute error are $0.46 \mathrm{~mm}$.

\section{DISCUSSION}

An RKPM-based meshfree method was proposed for reconstructing the deformation and stress field during needle insertion. The tissue crack and viscoelasticity of soft tissue have been integrated into the meshfree method. The traction/needle insertion force and the displacement boundaries are given by the force sensor and the needle insertion depth on every iteration to adapt the automatic computation requirement during the needle insertion procedure. To verify the accuracy of the proposed method, an experiment involving needle insertion into a silicon model was performed. The movements of 18 metallic beads inside the silicone model were recorded by the $\mathrm{X}$-ray images and be compared with the reconstructed results.

The comparison between the experimental measurements and the reconstructed displacements indicates that the proposed method has a comparable accuracy reported in the previous literature. The needle is inserted into the silicon model as deep as $40 \mathrm{~mm}$ which can cover the needle insertion procedures in abdominal organs. As we know, no work has compared the reconstructed displacement with the experimental measurement in such insertion depth and large deformation. Roberson et al. [3], [38] reported that an average error in radioactive seed placement was $1.2 \mathrm{~mm}$ for both seed types 

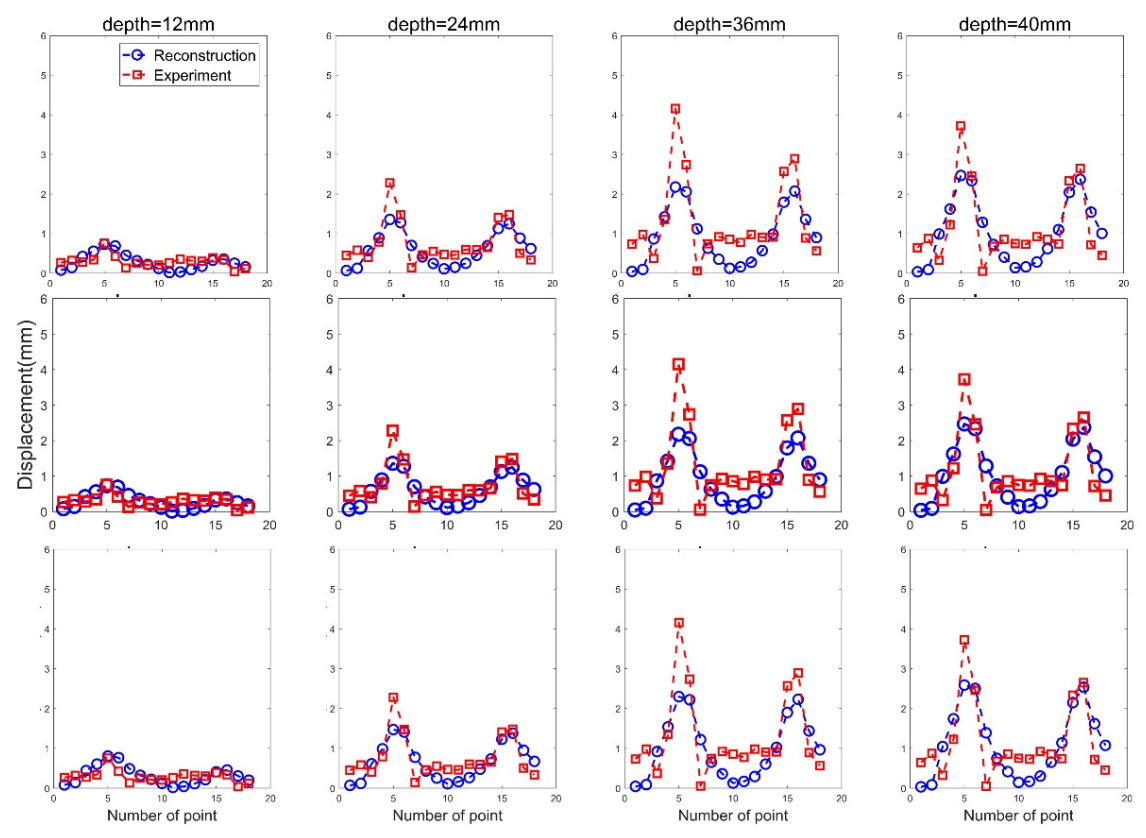

Figure 3: Comparison of reconstructed node displacement and experimental measurements at four different insertion depths during needle insertion experiment. The three rows of pictures represent respectively the results using 836,1881 , and 3344 nodes in reconstruction.

and the maximum error in seed placement was $2.1 \mathrm{~mm}$. In here, the total average error for the reconstructed displacement data in all insertion depths is smaller than the $1 \mathrm{~mm}$ which indicates that the reconstructed results are acceptable as it has lower error than the manually generated displacement error.

The decrease of the displacement reconstruction accuracy may be caused by the stress relaxation which existed both during and after the needle insertion. Stress relaxation is the viscoelastic characteristic of silicone gel. The specific manifestation of this characteristic is that the metallic beads rebound against the stretched direction during and after the needle insertion. From experimental measures of metallic beads during the needle insertion process, the displacements of all metallic beads start to decrease when needle insertion depth varies from $36 \mathrm{~mm}$ to $40 \mathrm{~mm}$ (Figure 6A). The average values of the normalized displacements of all metallic beads in Figure $6 \mathrm{~B}$ clearly show the decrease of the normalized displacement from 0.98 to 0.87 . After the needle stopped insertion (needle insertion stopped at the 20s), the silicon model continued to rebound back. Figure 7 shows four X-ray images respectively at the 20s, 25 s, $29 \mathrm{~s}, 34 \mathrm{~s}$. From the contours of the silicone model in the four pictures, it can be found that the silicon was constantly rebounding upwards which proves the existence of stress relaxation.

Assuming the stress relaxation of the silicon model can be described by the standard linear solid relaxation function [39], hence for maintaining a deformation $l(t)$, the maintaining force is expressed as:

$$
F(t)=E_{R}\left[1-\left(1-\frac{\tau_{\sigma}}{\tau_{\varepsilon}}\right) e^{-t / \tau_{\varepsilon}}\right] l(t)
$$

where $E_{R}$ is the relaxed elastic modulus, $\tau_{\sigma}$ is the relaxation time of elongation under the condition of constant load, $\tau_{\epsilon}$ is the relaxation time of load under the condition of constant elongation. If the friction force applied on the silicon model $f(t)$ is smaller than the $F(t)$ (as presented in Figure 6), the deformation will creep back as the friction force cannot maintain the deformation. The decrease in the displacement of metallic beads caused by the stress relaxation may be an important reason for explaining the difference between the reconstructed displacements and experimental measurements. As the stress relaxation happened during the needle insertion with constant velocity, it can be avoided by accelerating the needle insertion speed.

\section{CONCLUSIONS}

We have presented an RKPM-based meshfree method for automatically reconstructing the deformation and stress field inside the soft tissue during needle insertion. The proposed method has considered the crack and viscoelasticity of soft tissue which is an organ-like material. The meshfree method does not require manually updating the model mesh during the crack and the boundary conditions including the needle insertion force and the needle tip position can be provided by the motion control system of needle insertion robotic in a real-time manner. Therefore, the whole computation process of reconstruction can be automatic during the needle insertion procedure.

The method has been validated by the experiment involving needle insertion into the silicon phantom using the robotic arm. The comparison between the reconstructed data and experimental measurements indicates the accuracy is acceptable during large 

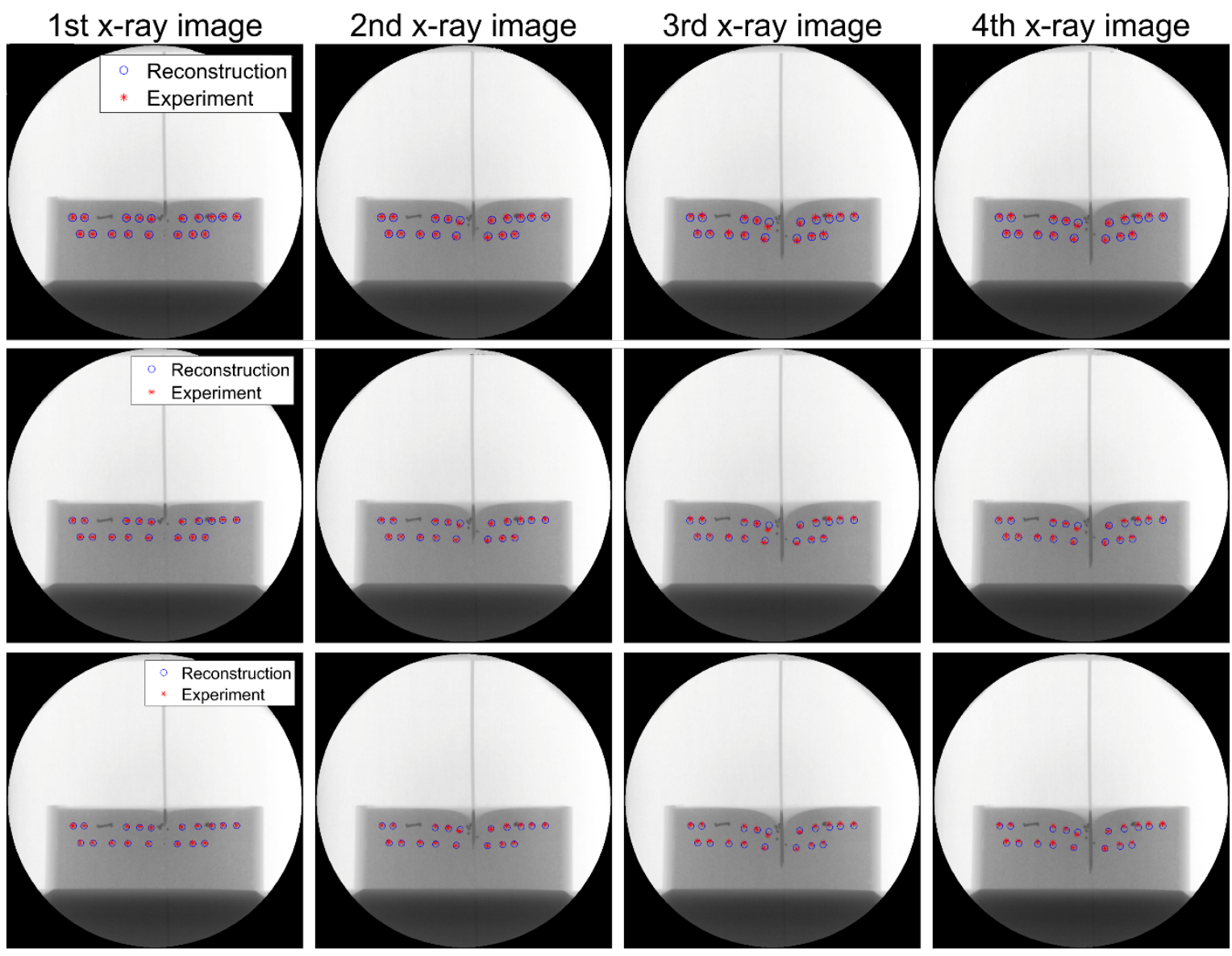

Figure 4: Comparison of the reconstructed displacement of metallic beads registered to the $\mathrm{X}$-ray images during the needle insertion experiment. The three rows of pictures represent respectively the results using 836,1881 , and 3344 nodes.
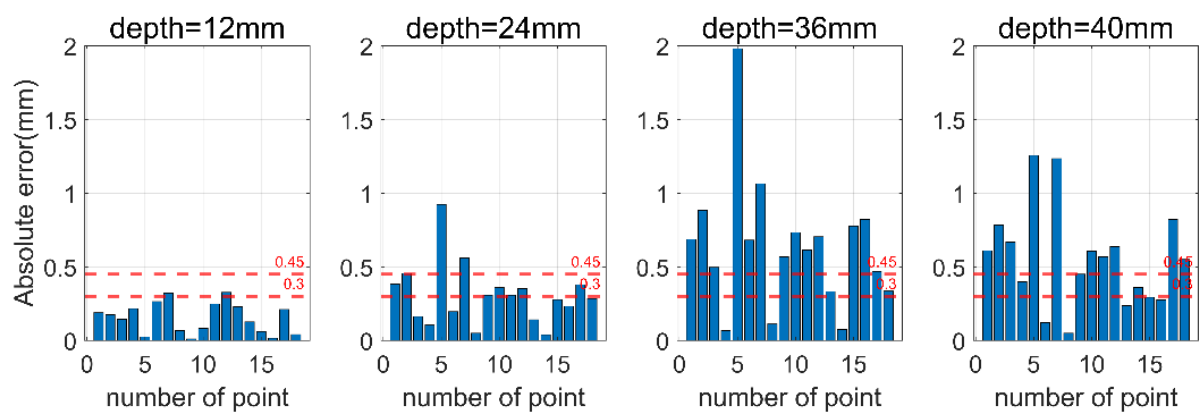

Figure 5: Absolute error of the reconstructed displacements from the experimental measurements using 1881 nodes. The red lines are two accuracy thresholds: $0.3 \mathrm{~mm}$ and $0.45 \mathrm{~mm}$.

deformation. As the insertion depth increase, the stress relaxation will increase the reconstruction error. Although both silicon model and human soft organ, such as the liver, have similar elasticity and viscoelasticity, further study should focus on the influence of stress relaxation in a real human organ in future work. The proposed method can be used to automatically predict and track the needle insertion target during operation which increases the operation accuracy. 

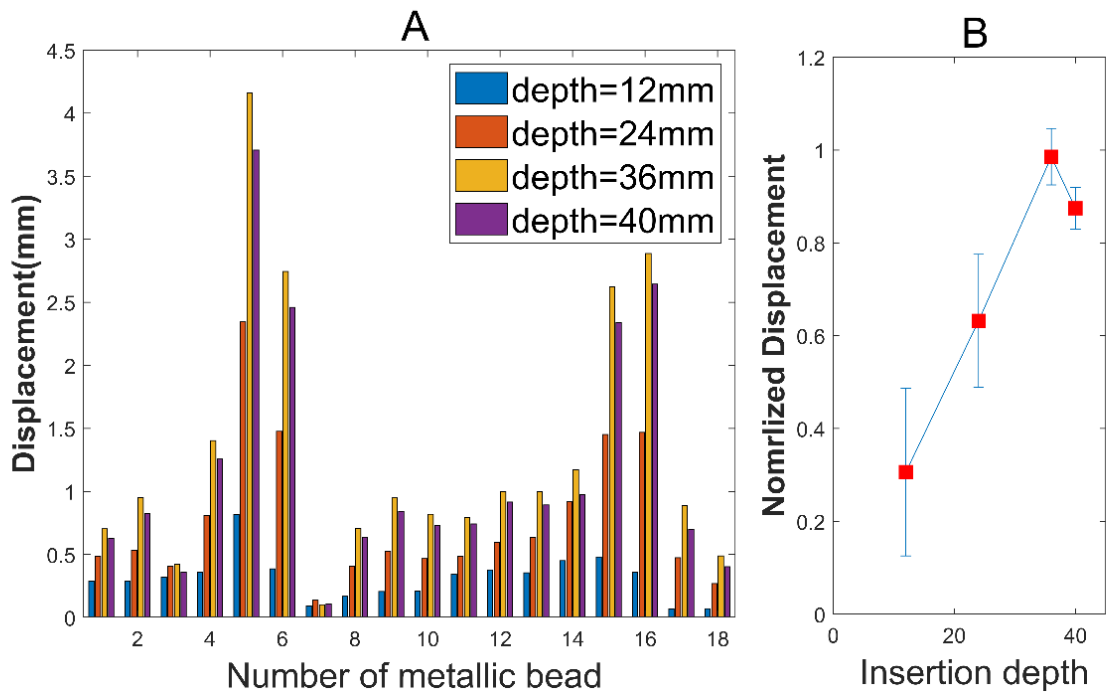

Figure 6: Stress relaxation of metallic beads during needle insertion. A. The displacement of metallic beads at different insertion depths; B. Average and standard deviation of normalized displacement of all metallic beads at four different insertion depths.
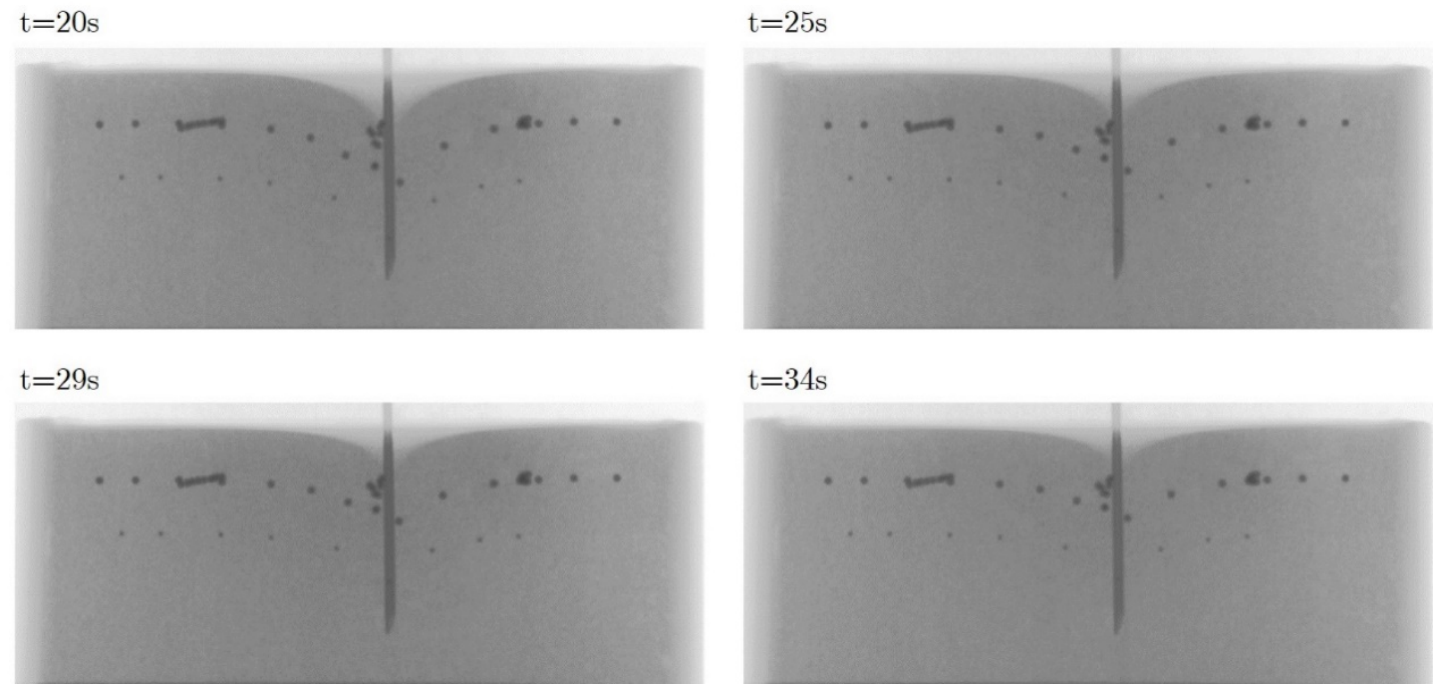

Figure 7: Four X-ray images respectively at the $20 s, 25 s, 29 s, 34 s$ when the stress relaxation still occurs even if the needle is stopped.

\section{ACKNOWLEDGMENTS}

This study was financed partially by the National Key R\&D Program of China (No.2018YFA0704102), the National Natural Science Foundation of China (No.81827805), Shenzhen Key R\&D Program (No.JCYJ2020019114812361), Shenzhen Key R\&D Program (No.JCYJ20200109114610201), China Postdoctoral Science Foundation (No. 2020M682960) and the SIAT Innovation Program for Excellent Young Researchers.

\section{REFERENCES}

[1] N. Abolhassani, R. Patel, and M. Moallem, "Needle insertion into soft tissue: A survey," Medical Engineering \& Physics, vol. 29, no. 4, pp. 413-431, May 2007, doi: 10.1016/j.medengphy.2006.07.003.

[2] M. Mahvash and P. E. Dupont, "Mechanics of Dynamic Needle Insertion into a Biological Material," IEEE Transactions on Biomedical Engineering, vol. 57, no. 4, pp. 934-943, Apr. 2010, doi: 10.1109/TBME.2009.2036856.

[3] A. K. H. Robertson, P. S. Basran, S. D. Thomas, and D. Wells, "CT, MR, and ultrasound image artifacts from prostate brachytherapy seed implants: The impact of seed size," Medical Physics, vol. 39, no. 4, pp. 2061-2068, 2012, doi: 10.1118/1.3694669.

[4] A. Leibinger, M. J. Oldfield, and F. Rodriguez y Baena, "Minimally disruptive needle insertion: a biologically inspired solution," Interface Focus, vol. 6, no. 3, p. 20150107, Jun. 2016, doi: 10.1098/rsfs.2015.0107. 
[5] A. D. R. Li, K. B. Putra, L. Chen, J. S. Montgomery, and A. Shih, "Mosquito proboscis-inspired needle insertion to reduce tissue deformation and organ displacement," Scientific Reports, vol. 10, no. 1, Dec. 2020, doi: 10.1038/s41598-02068596-w.

[6] T.-N. Nguyen, M.-C. Ho Ba Tho, and T.-T. Dao, "A Systematic Review of Real-Time Medical Simulations with Soft-Tissue Deformation: Computational Approaches, Interaction Devices, System Architectures, and Clinical Validations," Appl Bionics Biomech, vol. 2020, Feb. 2020, doi: 10.1155/2020/5039329.

[7] G. Joldes et al., "Suite of meshless algorithms for accurate computation of soft tissue deformation for surgical simulation," Medical Image Analysis, vol. 56, pp 152-171, Aug. 2019, doi: 10.1016/j.media.2019.06.004.

[8] R. Plantefève, I. Peterlik, N. Haouchine, and S. Cotin, "Patient-Specific Biomechanical Modeling for Guidance During Minimally-Invasive Hepatic Surgery," Ann Biomed Eng, vol. 44, no. 1, pp. 139-153, Jan. 2016, doi: 10.1007/s10439-015-1419-z

[9] K. Liang, M. Ruess, and M. Abdalla, "Co-rotational finite element formulation used in the Koiter-Newton method for nonlinear buckling analyses," Finite Elements in Analysis and Design, vol. 116, pp. 38-54, Sep. 2016, doi: 10.1016/j.finel.2016.03.006.

[10] K. Miller, G. Joldes, D. Lance, and A. Wittek, "Total Lagrangian explicit dynamics finite element algorithm for computing soft tissue deformation," Communications in Numerical Methods in Engineering, vol. 23, no. 2, pp. 121-134, 2007, doi: $10.1002 / \mathrm{cnm} .887$.

[11] F. Meister, T. Passerini, V. Mihalef, A. Tuysuzoglu, A. Maier, and T. Mansi, "Deep learning acceleration of Total Lagrangian Explicit Dynamics for soft tissue mechanics," Computer Methods in Applied Mechanics and Engineering, vol. 358, p 112628, Jan. 2020, doi: 10.1016/j.cma.2019.112628

[12] K. Vemaganti, G. R. Joldes, K. Miller, and A. Wittek, "Total Lagrangian Explici Dynamics-Based Simulation of Tissue Tearing," in Computational Biomechanics for Medicine, New York, NY, 2011, pp. 63-72. doi: 10.1007/978-1-4419-9619-0_7.

[13] M. Bucki, C. Lobos, and Y. Payan, "A fast and robust patient specific Finite Element mesh registration technique: Application to 60 clinical cases," Medical Image Analysis, vol. 14, no. 3, pp. 303-317, Jun. 2010, doi: 10.1016/j.media.2010.02.003.

[14] P. Lamata et al., "An accurate, fast and robust method to generate patient-specific cubic Hermite meshes," Medical Image Analysis, vol. 15, no. 6, pp. 801-813, Dec. 2011, doi: 10.1016/j.media.2011.06.010.

[15] J.-S. Chen, M. Hillman, and S.-W. Chi, "Meshfree Methods: Progress Made after 20 Years," Journal of Engineering Mechanics, vol. 143, no. 4, p. 04017001, Apr. 2017, doi: 10.1061/(ASCE)EM.1943-7889.0001176.

[16] "A two-level strain smoothing regularized meshfree approach with stabilized conforming nodal integration for elastic damage analysis - Dongdong Wang, Zhuoya Li, 2013." https://journals.sagepub.com/doi/10.1177/1056789512455938 (accessed Oct. 16, 2020).

[17] M. K. Rausch, G. E. Karniadakis, and J. D. Humphrey, "Modeling Soft Tissue Damage and Failure Using a Combined Particle/Continuum Approach," Biomech Model Mechanobiol, vol. 16, no. 1, pp. 249-261, Feb. 2017, doi: 10.1007/s10237-0160814-1.

[18] Y. Wu, D. Wang, C.-T. Wu, and H. Zhang, "A direct displacement smoothing meshfree particle formulation for impact failure modeling," International Journal of Impact Engineering, vol. 87, pp. 169-185, Jan. 2016, doi: 10.1016/j.ijimpeng.2015.03.013.

[19] F. Amiri, D. Millán, M. Arroyo, M. Silani, and T. Rabczuk, "Fourth order phase-field model for local max-ent approximants applied to crack propagation," Computer Methods in Applied Mechanics and Engineering, vol. 312, pp. 254-275, Dec. 2016, doi: 10.1016/j.cma.2016.02.011

[20] B. Nayroles, G. Touzot, and P. Villon, "Generalizing the finite element method: Diffuse approximation and diffuse elements," Computational Mechanics, vol. 10, no. 5, pp. 307-318, Sep. 1992, doi: 10.1007/BF00364252.

[21] T. Belytschko, Y. Y. Lu, and L. Gu, "Element-free Galerkin methods," International Journal for Numerical Methods in Engineering, vol. 37, no. 2, pp. 229-256, 1994 doi: 10.1002/nme.1620370205.
[22] T. Belytschko, Y. Krongauz, D. Organ, M. Fleming, and P. Krysl, "Meshless methods: An overview and recent developments," Computer Methods in Applied Mechanics and Engineering, vol. 139, no. 1, pp. 3-47, Dec. 1996, doi: 10.1016/S00457825(96)01078-X

[23] W. K. Liu, S. Jun, and Y. F. Zhang, "Reproducing kernel particle methods," International Fournal for Numerical Methods in Fluids, vol. 20, no. 8-9, pp. 1081-1106, 1995, doi: 10.1002/fld.1650200824.

[24] C. A. Duarte and J. T. Oden, "An h-p adaptive method using clouds," Computer Methods in Applied Mechanics and Engineering, vol. 139, no. 1, pp. 237-262, Dec. 1996, doi: 10.1016/S0045-7825(96)01085-7.

[25] I. Babuška and J. M. Melenk, "The Partition of Unity Method," International fournal for Numerical Methods in Engineering, vol. 40, no. 4, pp. 727-758, 1997, doi: 10.1002/(SICI)1097-0207(19970228)40:4<727::AID-NME86>3.0.CO;2-N.

[26] E. J. Kansa, "Multiquadrics-A scattered data approximation scheme with applications to computational fluid-dynamics-I surface approximations and partial derivative estimates," Computers \& Mathematics with Applications, vol. 19, no. 8, pp. 127-145, Jan. 1990, doi: 10.1016/0898-1221(90)90270-T.

[27] X. Zhang, K. Z. Song, M. W. Lu, and X. Liu, "Meshless methods based on collocation with radial basis functions," Computational Mechanics, vol. 26, no. 4, pp. cation with radial basis functions,' Computational

[28] R. Vertnik and B. Šarler, "Meshless local radial basis function collocation method for convective-diffusive solid-liquid phase change problems," International fournal of Numerical Methods for Heat \& Fluid Flow, vol. 16, no. 5, pp. 617-640, Jan. 2006, doi: 10.1108/09615530610669148.

[29] J. S. Chen, W. Hu, and H. Y. Hu, "Reproducing kernel enhanced local radial basis collocation method," International fournal for Numerical Methods in Engineering, vol. 75 , no. 5 , pp. 600-627, 2008, doi: 10.1002/nme.2269.

[30] E. Oñate, S. Idelsohn, O. C. Zienkiewicz, and R. L. Taylor, "A Finite Point Method in Computational Mechanics. Applications to Convective Transport and Fluid Flow," International fournal for Numerical Methods in Engineering, vol. 39, no. 22, pp. 3839-3866, 1996, doi: 10.1002/(SICI)1097-0207(19961130)39:22<3839::AIDNME27>3.0.CO;2-R.

[31] D. Wang, J. Wang, and J. Wu, "Superconvergent gradient smoothing meshfree collocation method," Computer Methods in Applied Mechanics and Engineering, vol. 340, pp. 728-766, Oct. 2018, doi: 10.1016/j.cma.2018.06.021.

[32] A. Horton, A. Wittek, G. R. Joldes, and K. Miller, "A meshless Total Lagrangian explicit dynamics algorithm for surgical simulation," International fournal for Numerical Methods in Biomedical Engineering, vol. 26, no. 8, pp. 977-998, 2010, doi: $10.1002 / \mathrm{cnm} .1374$.

[33] H. Ahmadzadeh, M. K. Rausch, and J. D. Humphrey, "Particle-based computational modelling of arterial disease," f R Soc Interface, vol. 15, no. 149, Dec. 2018, doi: 10.1098/rsif.2018.0616.

[34] J. Hong, T. Dohi, M. Hashizume, K. Konishi, and N. Hata, "An ultrasound-driven needle-insertion robot for percutaneous cholecystostomy," Phys. Med. Biol., vol. 49, no. 3, pp. 441-455, Jan. 2004, doi: 10.1088/0031-9155/49/3/007.

[35] T. Rabczuk, S. Bordas, and G. Zi, "On three-dimensional modelling of crack growth using partition of unity methods," Computers \& Structures, vol. 88, no. 23, pp. 1391-1411, Dec. 2010, doi: 10.1016/j.compstruc.2008.08.010.

[36] X. Jin, G. R. Joldes, K. Miller, K. H. Yang, and A. Wittek, "Meshless algorithm for soft tissue cutting in surgical simulation," Computer Methods in Biomechanics and Biomedical Engineering, vol. 17, no. 7, pp. 800-811, May 2012, doi: 10.1080/10255842.2012.716829.

[37] A. Wittek, G. Bourantas, B. F. Zwick, G. Joldes, L. Esteban, and K. Miller, "Mathematical Modeling and Computer Simulation of Needle Insertion into Soft Tissue," arXiv:2002.08123 [physics], Feb. 2020, Accessed: Jun. 15, 2020. [Online]. Available: http://arxiv.org/abs/2002.08123

[38] P. L. Roberson, V. Narayana, D. L. McShan, R. J. Winfield, and P. W. McLaughlin, "Source placement error for permanent implant of the prostate," Medical Physics, vol. 24 , no. 2, pp. 251-257, 1997, doi: 10.1118/1.598058.

[39] Y. C. Fung, "Comparison of different models of the heart muscle," fournal of Biomechanics, vol. 4, no. 4, pp. 289-295, Jul. 1971, doi: 10.1016/0021-9290(71)90035- 